To follow the dynamics of cohesin in response to a DSB in the budding yeast Saccharomyces cerevisiae, Ünal et al. ${ }^{1}$ and Ström et al. ${ }^{2}$ have used a well-characterized system in which a DSB can be generated at a defined point of the genome, by inducing the expression of a specific DNA-cutting enzyme called HO endonuclease. By using a highresolution mapping technique - chromatin immunoprecipitation - both groups find that cohesin accumulates in a large chromosomal region of around 50-100 kilobases surrounding the DSB. Moreover, this local enrichment of cohesin at the DSB site occurs in G2 phase and requires the same protein (Scc2) that facilitates genome-wide loading of cohesin in early $S$ phase. These observations strongly suggest that the DSB induces de novo loading of cohesin at the damaged site, rather than a rearrangement of cohesin already loaded on the DNA during S phase.

What is the molecular mechanism underlying this local recruitment of cohesin? A previous study ${ }^{7}$ showed that $\mathrm{H} 2 \mathrm{AX}-\mathrm{a}$ histone protein, involved in packaging DNA - is phosphorylated in a large region that extends some 50 kilobases either side of an HO-endonuclease-induced DSB in yeast. This modification depends on two enzymes - namely Tell and Mec1 (ATM and ATR, respectively, in mammals) which are involved in a 'checkpoint' that delays cell division until damaged DNA is repaired ${ }^{7}$.

Following on from these findings, Ünal et al. ${ }^{1}$ show that cohesin recruitment in response to a DSB is also regulated by Tel1 and Mec1, and requires H2AX phosphorylation. The formation of the region of phosphorylated H2AX proteins is therefore likely to be a prerequisite for the loading of cohesin (Fig. 1). Equally important, Ström et al. ${ }^{2}$ provide evidence that the DSB-induced cohesin loading does indeed establish a de novo linkage between the damaged chromatid and its undamaged sister, thereby facilitating DSB repair. Consistent with this conclusion, cohesin is not required for other repairrelated processes, such as intrachromosomal gene conversion or the resection of broken DNA strands ${ }^{1}$ — events that do not require a template or, therefore, new sister-chromatid linkages.

These observations made in yeast are likely to be highly relevant to our understanding of DSB repair in mammals. In fact, DNA-damage-induced recruitment of cohesin was first hinted at through a lowerresolution, cytological method - immunofluorescent microscopy - in human cells ${ }^{8}$. This recruitment was dependent on the DSB-repair protein Mre11, as has been confirmed in yeast ${ }^{1}$. Moreover, other studies showed that ATM phosphorylates one component of cohesin following DNA damage induced by ionizing irradiation, and that this phosphorylation is required for activation of the DNA-damage checkpoint ${ }^{9,10}$. Cells expressing only a non-phosphorylatable form of cohesin support normal mitotic progression, but exhibit a defective checkpoint response and increased chromosomal aberrations ${ }^{11}$, implying that this modification of cohesin is specialized for DNA repair in mammalian cells.
In summary, the new studies ${ }^{1,2}$ underscore the importance of a cohesin-mediated sister linkage in DSB repair. They also provide a fresh view of cohesin - which seems to be much more dynamic than had been thought - and raise several new questions. What is the real signal or modification that allows cohesin to accumulate at the DSB site? Does cohesin, or the protein that loads it onto DNA, interact directly with phosphorylated $\mathrm{H} 2 \mathrm{AX}$ ? What happens when DNA repair is completed? Might cohesin need to be cleaved in order to be unloaded after DNA repair ${ }^{12}$, as has been shown in mitosis ${ }^{3}$ ? Undoubtedly, answering these questions will further enrich our understanding of the global and local dynamics of chromosome architecture, and its impact on genome stability.

Tatsuya Hirano is at the Cold Spring Harbor Laboratory, One Bungtown Road, Cold Spring Harbor, New York 11724, USA.

e-mail:hirano@cshl.edu

1. UUnal, E. et al. Mol. Cell 16, 991-1002 (2004).

2. Ström, L., Lindroos, H. B., Shirahige, K. \& Sjögren, C. Mol. Cell 16, 1003-1015 (2004).

3. Nasmyth, K. Annu. Rev. Genet. 35, 673-745 (2001).

4. Birkenbihl, R. P. \& Subramani, S. Nucl. Acids Res. 20, 6605-6611 (1992).

5. Sjögren, C. \& Nasmyth, K. Curr. Biol. 11, 991-995 (2001).

6. Sonoda, E. et al. Dev. Cell 1, 759-770 (2001)

7. Shroff, R. et al. Curr. Biol. 14, 1703-1711 (2004).

8. Kim, J. S., Krasieva, T. B., LaMorte, V., Taylor, A. M.

\& Yokomori, K. J. Biol. Chem. 277, 45149-45153

(2002).

9. Kim, S.-T., Xu, B. \& Kastan, M. B. Genes Dev. 16, 560-570 (2002).

10. Yazdi, P. T. et al. Genes Dev. 16, 571-582 (2002).

11. Kitagawa, R., Bakkenist, C. J., McKinnon, P. J. \& Kastan, M. B. Genes Dev. 18, 1423-1438 (2004).

12. Nagao, K., Adachi, Y. \& Yanagida, M. Nature 430, 1044-1048 (2004).

Mycology

\title{
To be or not to be a lichen
}

Work by Mats Wedin and colleagues highlights both the versatility of fungi and the complications these unsung organisms pose for the biologist. Two of the ways in which fungi make a living are as saprophytes, drawing sustenance from decaying matter, and as lichens, in which they form an intimate relationship with green algae or photosynthetic bacteria. Evidently, however, a single fungal species can adopt either lifestyle according to circumstance (New Phytol. 164, 459-465; 2004).

Using molecular techniques, Wedin et al. looked at fungi living on different parts of aspen (Populus tremula) in northern Sweden. On analysing four independent genomic markers, they found that three different species of a fungal genus (Stictis) grew directly on wood without bark as typical saprophytes (left photograph); but when on the bark of the trunk, the same species associated with green algae to form a whitish, crust-like lichen (right). In both images the scale bar is $1 \mathrm{~mm}$.

The lichens had hitherto been placed in a separate genus, Conotrema. Thirty-five years ago it was realized that the fruit bodies of Stictis and Conotrema are microscopically almost indistinguishable, but Stictis was not previously recognized as a lichen-forming genus. That species classified in separate genera are in fact the same organism exhibiting different biologies is a startling discovery. In all three cases, the wood-inhabiting non-lichenized and lichenized specimens were mixed together in phylogenetic trees.

Wedin et al. point out that the

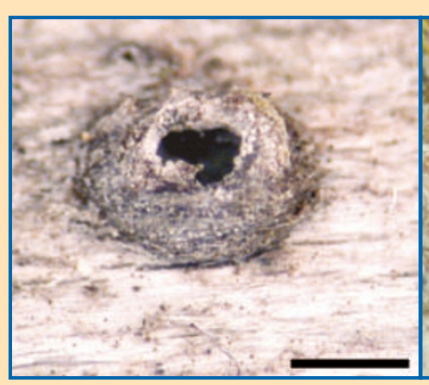

ability of a fungus to opt for different nutritional modes means that individual species can exploit a wider range of habitats. If the spores that are shot from the fruits of these fungi land on wood, they establish themselves as saprophytes; if they land on bark where appropriate algae are present, they form lichens.

The frequency of this plasticity in lifestyles is unknown. But it may be common, at least in this group of fungi, as the three species able to become optionally lichenized were found on a single species of tree.

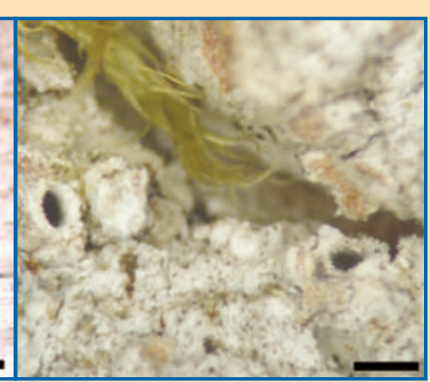

The study reinforces the case for never treating lichens as anything other than a lifestyle category among the fungi. It also shows the wisdom of the 1959 decision to restrict the scientific names of lichens to the fungal partners; in consequence, the same names can be used for the fungi regardless of their lifestyle. David Hawksworth David Hawksworth is in the Facultad de Farmacia, Universidad Complutense de Madrid, Plaza Ramón y Cajal s/n, 28040 Madrid, Spain.

e-mail:myconova@terra.es 